Cahiers $d u$ MONDE RUSSE

\section{Cahiers du monde russe}

Russie - Empire russe - Union soviétique et États indépendants

$60 / 4 \mid 2019$

Varia

\title{
Nicholas B. BREYFOGLE, ed., Eurasian Environments. Nature and Ecology in Imperial Russian and Soviet History
}

\section{Marin Coudreau}

\section{(2) OpenEdition}

1 Journals

Édition électronique

URL : https://journals.openedition.org/monderusse/11483

DOI : $10.4000 /$ monderusse. 11483

ISSN : $1777-5388$

Éditeur

Éditions de l'EHESS

Édition imprimée

Date de publication : 1 octobre 2019

Pagination : 823-830

ISBN : 978-2-7132-2797-4

ISSN : $1252-6576$

\section{Référence électronique}

Marin Coudreau, « Nicholas B. BREYFOGLE, ed., Eurasian Environments. Nature and Ecology in Imperial Russian and Soviet History », Cahiers du monde russe [En ligne], 60/4 | 2019, mis en ligne le 01 octobre 2019, consulté le 04 janvier 2023. URL : http://journals.openedition.org/monderusse/11483 ; DOI : https://doi.org/10.4000/monderusse.11483 


$\begin{array}{cccccccccccccccccccc}P & E & R & I & O & D & E & & S & O & V & I & E & T & I & Q & U & E \\ & E & T & & P & O & S & T & S & O & V & I & E & T & I & Q & U & E\end{array}$

Nicholas B. BREYFOGLE, ed.

\section{Eurasian Environments}

Nature and Ecology in Imperial Russian and Soviet History

Pittsburgh : University of Pittsburg Press, 2018, 424 p.

Eurasian Environments vient combler un manque historiographique important en proposant un ensemble de cas d'études en histoire environnementale des régions eurasiatiques, soit une part non négligeable de la surface de la planète, qui occupe pourtant encore une place relativement marginale dans la sphère des études environnementales. Comme le rappelle Nicholas Breyfogle dans son introduction, le champ s'est développé aux États-Unis dans les années 1970 et n'a commencé à peser dans l'historiographie de la Russie impériale, soviétique et post-soviétique que récemment. Après avoir indiqué quelques principes donnant corps à ce domaine d'étude, « une histoire rarement simplement humaine » (p. 7), Breyfogle s'attache à montrer l'importance de la Russie et de l'Union soviétique pour le champ de l'histoire environnementale, et vice-versa, avant d'ouvrir sur les perspectives d'une telle histoire pour notre compréhension des enjeux stratégiques et climatiques actuels. L'ouvrage se déploie ensuite sur cinq parties qui font la part belle aux milieux : 1. «L'environnement des steppes »; 2. «L'ingénierie hydraulique »; 3. « La terre, les roches, le sol»; 4. « Les fruits de l'eau »; 5. « Corps et maladies, santé et environnement ».

David Moon ouvre la première partie des contributions en s'intéressant au travail de Sisyphe que furent les tentatives de plantation d'arbres dans le milieu sec des steppes. L'absence d'arbres était aussi considérée comme « une faute requérant correction » par les colons slaves et germaniques (p. 30). Moon met en lumière combien la présence de forêts était associée à la culture (européenne), et leur absence (voire leur destruction supposée), à l'arriération des autochtones, une conception que l'auteur met au diapason des pratiques coloniales européennes. Ainsi pour Moon, les plantations d'arbres dans les steppes s'inscrivaient plus largement dans les pratiques racontées par Alfred Crosby où les colons Européens cherchaient à transformer, avec leurs plantes, leurs animaux et leurs maladies, les parties tempérées du globe en « néo-Europes ». Contrairement aux entreprises coloniales européennes toutefois, les colons qui gagnèrent les steppes en essayant de « recréer des paysages forestiers familiers, rencontrèrent de sérieuses difficultés, car l'environnement des steppes n'était pas apte au boisement» (p. 32-33).

Sarah Cameron analyse pour sa part l'évolution des steppes kazakhes à la fin de la période tsariste pour déceler les héritages impériaux de la grande famine déclenchée par la collectivisation forcée, l'objet d'étude au cœur de son travail de thèse. « Bien que l'assaut de Moscou contre la campagne fût la première cause de la famine kazakhe, les legs du pouvoir impérial russe doivent être considérés comme un facteur important » (p. 44). Après avoir dessiné en détail les écologies et les 
relations intriquées entre les steppes et les nomades kazakhes, Cameron raconte les effets écologiques des colons, désignés par les Kazakhs comme « ceux qui sont venus sans invitation ». Cameron note que depuis Catherine II, le commerce des céréales devait " aider à " civiliser » les Kazakhs et à en faire des sujets loyaux de l'empire ». Entre 1896 et 1916, une période d'intensification de la colonisation, « les autorités continuèrent à lier la consommation du grain à l'adoption de la vie sédentaire » (p. 55). En étudiant les impacts de la colonisation de près d'un million et demi de paysans russes à la fin de l'époque tsariste, Cameron décèle des processus de fragilisation des populations nomades à l'œuvre dans l'altération de leurs environnements (pression accrue sur les ressources, développement d'épizooties) sans toutefois que « la disparition de la vie nomade sous le régime soviétique soit préétablie ou inévitable » (p. 58).

Phénomène lié aux aléas climatiques et pouvant engendrer la mort du bétail, la perte de fourrage par le gel des pâtures (žut) au Kazakhstan est au centre du cas d'étude de Ian W. Campbell. Le žut, qui survenait environ tous les dix ou douze ans, était perçu par les nomades comme une « expérience déplaisante » plutôt qu'une catastrophe, du fait de leur mobilité (p. 62). Les restrictions aux migrations créées par les frontières et la colonisation russe ont cependant affaibli les possibilités de fuite des nomades face au žut. À partir des années 1860, cet événement naturel constitua, pour les autorités impériales, « un marqueur clair à l'instabilité inhérente au mode de vie du nomadisme pastoral » (p. 73). L'agriculture était considérée comme « le moyen le plus favorable et prééminent à la lutte contre le žut» (p. 67). De manière similaire au chapitre précédent, Campbell montre ainsi comment l'intervention impériale, comprise comme « mission civilisatrice » (p. 61), a contribué à déqualifier les savoirs traditionnels des populations nomades et ainsi engendré une série de fragilisations des populations face au žut.

Toujours avec une focale portée sur les steppes kazakhes, Marc Elie analyse de manière fouillée les liens complexes entre sécheresses, politiques agricoles, savoirs et changement climatiques dans les années de la grande Campagne des Terres Vierges (CTV) de Hruščev. Après avoir développé ses bases méthodologiques sur les fondations des disaster studies et de l'histoire environnementale, Elie retrace comment, en dépit des avancées russes des sciences climatiques et météorologiques, le « Plan pour la Transformation de la Nature » lancé par Stalin au lendemain de la seconde guerre mondiale a « favorisé la diffusion de conceptions erronées de la sécheresse » (p. 84) menant à d'immenses travaux d'infrastructures inefficaces (p. 85) développés sur le dos des paysans et des prisonniers du Goulag. $\mathrm{Au}$ lancement de la CTV, les autorités ne possèdent aucune donnée sur la sécheresse dans la région (à l'est de l'Oural) et il faudra attendre encore vingt ans pour que la première monographie sur la sécheresse soit enfin publiée au Kazakhstan (p. 85-87). Rejetant les explications officielles des mauvaises récoltes en termes de « catastrophes naturelles », Marc Elie conclut que « (l’) érosion provoquée par un mauvais traitement des sols explique les mauvaises récoltes, et les mauvaises décisions de Hruščev expliquent la crise alimentaire » (p. 87). La dernière séquence fait ressortir les enjeux complexes d'un panel de travaux de pédologues, 
météorologistes, climatologue et agronomes soviétiques émergeant dans les années 1970 autour des causes humaines et climatiques du « Dust Bowl soviétique », et qui s'inscrivent dans un même mouvement dans les préoccupations écologiques et scientifiques transnationales de la période.

Deux contributions se concentrent dans un deuxième temps sur les systèmes d'irrigation en Asie Centrale. Christian Teichmann décrit les défis posés dans l'entre-deux-guerres par le fleuve « capricieux » de l'Amu Dar'ja, région où l'irrigation « structurait l'ordre social des populations vivant le long du canal » (p. 101). L'auteur met ensuite en exergue les processus de « périphérisation » et de « marginalisation » de la région : par les délimitations nationales des républiques soviétiques en 1924-2025 qui divisent la région en trois unités administratives (créant de nouvelles tensions autour des ressources hydrauliques), mais aussi par le fait d'une dépréciation du modèle autochtone d'exploitation de l'eau par suite de la conquête bolchevique. Puis la collectivisation forcée dans cette localité est analysée au prisme du concept de « despotisme administratif » générant une « administration locale fragile (mais violente), mais aussi une administration centrale fragile (mais violente) » (p. 110). Teichmann insiste ainsi sur le fait que les agents de l'État se révélaient « incapables d'obtenir le contrôle du système d'irrigation », et que la construction des ouvrages hydrauliques reposait « bien plus sur l'improvisation que sur la planification $\gg$ (p. 110-112).

Julia Obertreis étudie l'émergence de critiques écologiques autour des questions de l'envasement de la mer d'Aral et de détournement de fleuves pour la culture du coton à partir des années 1970. L'auteure perçoit dans l'année 1975 une « césure » à partir de laquelle plusieurs lignes de « protection de la nature » sont catalysées par une série d'acteurs d'une sphère publique « fragmentée », et au diapason de tendances « globales » (p. 119-122). Les années de la perestroïka constituent un moment charnière du développement de l'environnementalisme en Asie centrale. Scientifiques et journalistes intensifièrent alors leurs discours critiques pour attirer l'attention du public sur les problèmes notamment liés à la pollution et à la disparition de l'eau (p. 123). L'auteur insiste sur le caractère pansoviétique, non ethnique, de ces critiques écologiques formulées par les élites d'Asie centrale et de Moscou (p. 123-125). Pour Obertreis, l'environnementalisme développé dans les pays socialistes représente « un type distinct » bien qu'inscrit toutefois dans un spectrum environnementaliste plus large (p. 128).

La troisième partie du livre s'ouvre sur une enquête originale de Mieka Erley consacrée à la réception du travail du chimiste Justus Liebig en Russie. Erley décrit comment la philosophie matérialiste, symbiotiquement liée au radicalisme politique d'une génération militante surgissant dans les années 1860, a constitué un terreau favorable à la réception des travaux de Liebig et à leur coloration politique. « En Europe, les travaux de Liebig émergèrent dans une période de révolution politique, mais en Russie, ces travaux furent reçus dans une ère de répression politique » (p. 138). Erley montre en effet que les théories de Liebig « offraient un moyen de parler des nouvelles philosophies matérialistes et de critiquer l'ordre sociopolitique russe ancré dans une économie du sol déséquilibrée » (p. 139). En étudiant les références 
à Liebig et ses travaux dans la littérature et dans les écrits des penseurs radicaux (de Marx à Lenin, en passant par Černyševskij et Engel'gardt), Mieka Erley trace les transferts métaphoriques réalisés entre un modèle de sol élaboré par le chimiste allemand et un modèle d'organisation sociale (critiqué ou souhaité). Elle perçoit ainsi une généalogie du concept soviétique de smyčka (désignant l'alliance entre la ville et la campagne) dans le système métabolique du sol de Liebig (p. 144-146).

S'appuyant sur un ensemble de travaux conceptuels prenant en compte le rôle de la matérialité et des non-humains dans l'histoire, Andy Bruno retrace l'influence de la nephéline - une roche présente dans les montagnes de Hibiny dans la péninsule de Kola - dans la transformation de l'économie et de l'écologie de cette région. En effet, pour Bruno « la nature joue un rôle productif dans le développement de schèmes modernes pour la transformer » (p. 152). L'enquête montre d'abord comment cette roche aux multiples usages industriels a transformé la péninsule de Kola, très clairsemée durant la période tsariste, en « l'une des régions les plus construites de l'Arctique au $\mathrm{XX}^{\mathrm{e}}$ siècle » sous le régime soviétique. Bruno montre par ailleurs comment les scientifiques au service du régime ont cherché à combiner productivisme et conservation (via le concept d' " utilisation complexe des ressources naturelles ») (p. 154-155). Il met ensuite en avant le rôle devenu négatif de la roche, comme un déchet contaminant les fleuves, les lacs, mais aussi l'air et les corps par les rejets industriels jusque dans les années 1990.

Pey-Yi Chu étudie l'évolution des rapports soviétiques au permafrost. Objet d'étude d'une commission ad hoc mise en place par l'Académie des sciences en 1929, le permafrost est d'abord un objet de " conquête ». Dans ce cadre, où se cristallise l'idée d'une « confrontation » guerrière avec la nature, Chu offre des lignes saisissantes sur l'histoire du terme contesté désignant le permafrost, večnaja merzlota (littéralement « congélation éternelle ») connotant des origines « géologiquement distantes » du phénomène (« le temps des mammouths »). Selon Chu, le terme de večnaja merzlota, avec l'idée d' « éternité » et de temporalité cyclique de la nature qui lui était attachée, entrait en contradiction avec la temporalité soviétique d'une marche en avant continue et irréversible vers le communisme. « En luttant contre la večnaja merzlota, conclut $\mathrm{Chu}$, la société soviétique luttait aussi contre un temps indiscipliné et spontané » (p. 173-174). Pey-Yi Chu montre ensuite comment, malgré la rhétorique de la conquête, le permafrost contraignit les Soviétiques à s'y adapter de trois manières différentes, en le préservant, en l'utilisant et en le générant.

Dans une quatrième partie, quatre études discutent la construction et l'utilisation des « ressources naturelles » océaniques dans le Nord et l'Extrême Orient russe. Stephen Brain s'attache à étudier les spécificités d'une « éthique environnementale chrétienne » des populations Pomor. Discutant un ensemble de travaux de spécialistes de l'environnementalisme chrétien d'un côté, et de la culture Pomor d'un autre, l'auteur qualifie l'éthique environnementale des Pomor comme un « mix unique d'animisme et de christianisme» (p. 193). L'historien déploie ensuite son étude en étayant le rejet Pomor des appels impériaux, puis bolcheviks, «à intensifier l'exploitation des ressources naturelles, en défendant à la place les méthodes traditionnelles qui correspondaient à leur compréhension de l'économie morale » (p. 195). Dans 
la pratique Pomor, les pêcheurs attendaient que les poissons viennent à eux, plutôt que de les chasser au large pour maximiser la capture ; Brain donne des exemples passionnants de ce rejet, qui s'exprime dans le refus des filets de pêche circulaires, des bateaux à moteur, ainsi que de la pêche du béluga, un « animal sacré » perçu comme un allié pour orienter les poissons vers les filets (et dont la consommation était taboue) (p. 196, 197 et 200). La nationalisation des pêcheries (collectivisation forcée), l'éviction brutale des petits propriétaires de bateaux (dékoulakisation), et la destruction des églises, ont fini par altérer profondément les pratiques de la pêche dans la région. Avec l'introduction de nouveaux acteurs, des flottes de bateaux motorisés et de quotas de pêche décuplés, " l'ancienne éthique environnementale Pomor a été déplacée avec succès, la méthode 'passive' d'attendre les dons de la nature a été remplacée par la mécanisation et l'industrialisation » (p. 203).

Avec des perspectives plus ancrées dans l'histoire des sciences et techniques, Julia Lajus propose une étude en plusieurs points complémentaire à celle de Brain. En partant d'une catastrophe qui emporta 25 bateaux de pêche en mer de Barents en 1894, Lajus retrace comment le comité visant à une gestion des risques qui en a émergé devint bientôt une plateforme dans laquelle les scientifiques furent invités à « améliorer la vie des Pomor en leur apportant le savoir sur les ressources halieutiques » (p. 206). Par un jeu d'alliances entre autorités impériales, zoologues et industriels, les discussions visant à répondre à la catastrophe font progressivement place à des projets de modernisation. Les expéditions scientifiques se donnent ainsi pour objectif de «montrer aux pêcheurs où ils pouvaient trouver le poisson » (p. 212), au large, en vue d'industrialiser la pratique des Pomor. En l'absence d'investissements de capitaux importants dans la région, ce sont avant tout les scientifiques qui sont à l'œuvre dans le processus de « construction » du poisson comme « ressource ». Dans l'un des développements les plus fascinants, Lajus montre comment les experts comparaient ressources minérales et animales : « les scientifiques, analyse l'historienne, ne voyaient pas de grande différence entre la pêche et les mines, se concentrant sur les ressources elles-mêmes et non sur les utilisateurs locaux des ressources » (p. 215-216). Julia Lajus ouvre enfin sur le devenir postrévolutionnaire de ces projets impériaux, rendus finalement utiles « lorsque l'État soviétique devint l'ultime actionnaire et utilisateur de ces ressources » (p. 220).

Mark Sokolsky porte son analyse sur les politiques impériale et soviétique de colonisation de la côte extrême-orientale du Primor'e, où le saumon, les algues et d'autres fruits de la mer constituent une part essentielle du régime alimentaire et de l'économie des groupes indigènes. Avec la colonisation de la fin du XIX ${ }^{\mathrm{e}}$ siècle, les fonctionnaires tsaristes regardaient " l'utilisation des ressources naturelles et les revendications territoriales » comme étant «étroitement liées » (p. 227). Au centre de l'étude foisonnante de Sokolsky se trouve ainsi « l'effort pour remplacer les pêcheurs et cueilleurs d'Asie de l'Est par des 'Russes' » avant et après 1917 (p. 225). La fin de l'époque impériale est marquée par une préoccupation grandissante des élites russes pour l'épuisement des ressources halieutiques dans la région, attribuée à la « prédation » des étrangers (Japonais, Chinois, Coréens) accusés par là même d'« exploiter » les populations indigènes. Les mêmes articulations entre 
conservation et xénophobie sont prolongées durant la période soviétique, avec une « coloration de conflit de classe » (p. 234). Les autorités soviétiques redéploient des techniques qui ont fait leurs preuves avant 1917 : colonisation dirigée par l'État et investissement de capitaux. La première vague de travailleurs soviétiques relocalisés en Extrême-Orient était toutefois composée plutôt de " pêcheurs de rivière ", ou même de « dockers », que de marins au long cours (p. 236).

Ryan Tucker Jones s'intéresse à la littérature russe de fiction concernant la chasse à la baleine. Il décèle deux motifs centraux dans un corpus qui va de la fin de la période impériale à la fin de l'Union soviétique : la baleine comme problème et attracteur d'étrangers et la lutte entre l'homme et le léviathan. Avant que ne se développe une véritable industrie russe de la baleine dans les années 1930, l'animal était avant tout perçu comme un problème, c'était alors « une créature de valeur, mais évasive " qui attirait les étrangers sur les côtes, " menaçant la souveraineté impériale et le bien-être des sujets indigènes » (p. 243). Alors que la littérature américaine « célébrait la croissance des villes autour de l'industrie de la baleine (...) les Russes impériaux voyaient les baleines au large de leurs côtes principalement comme des problèmes "), entraînant des étrangers venus détruire les richesses de la nature russe (p. 247). Tucker montre aussi comment A.A. Vakhov, écrivain à succès d'Exrême-Orient, et auteur d'une trilogie de fiction sur la chasse à la baleine dans l'après-1945, trace l'héritage d'une éthique environnementale russe dans l'apprentissage, auprès du peuple autochtone des Tchouktches, d'une sorte de chasse respectant la baleine opposée à la prédation américaine. Ces articulations fictives furent récupérées avec profit par les officiels soviétiques, notamment pour obtenir au niveau international une exception à l'interdiction de la chasse à la baleine grise pour les Tchouktches (p. 254).

Deux contributions consacrées aux questions de santé environnementale forment la dernière partie de cet ouvrage. George Lywood étudie les liens entre l'environnement de la côte criméenne, la littérature médicale et la perception publique de la région à la fin de la période tsariste. Malgré la révolution bactériologique, les médecins criméens de la fin du XIX ${ }^{\mathrm{e}}$ siècle adhéraient à « une vision avant tout environnementale des pathologies et de la médecine » et étaient partisans d'une « géographie médicale (ou topographie environnementale) » (p. 265-266). Après avoir énoncé comment les cures environnementales ont suscité une image publique de la région comme une " côte de santé ", Lywood livre une série d'informations sur les pratiques curatives de la côte criméenne, déclinées ainsi en « climatothérapie », " balnéothérapie », " thérapie solaire », « thérapie vinicole, lait et koumis » et « bains de boue et sanatoriums le long de la côte ». Les nombreux détails donnés par l'auteur permettent de connecter une série de données environnementales à des savoirs thérapeutiques produits alors par les médecins de la région. On apprend par exemple que la balnéothérapie était notamment conseillée pour guérir l' " efféminement » (iznežennost $t^{\prime}$ ) (p. 270) (et on notera aussi que c'est là la seule perspective sur le genre de tout l'ouvrage). Pour terminer, Lywood développe une comparaison intéressante avec l'Ouest américain du temps de Mark Twain, où, en 1900, un migrant sur quatre s'installait en Californie pour des raisons de santé (p. 278-279). 
La dernière contribution du livre proposée par Lisa $\mathrm{K}$. Walker porte sur la malaria et la lutte contre les moustiques au Tadjikistan dans l'entre-deux-guerres. L'auteur rappelle qu'après l'entrée de l'Armée rouge à Buhara en 1920, la prévalence de la malaria dans la région s'élève à $96 \%$, avec des taux de mortalité de sa forme tropicale atteignant les $30 \%$ (p. 281). La démonstration de Walker est centrée sur le passage d'un contrôle appréhendé en terme écologique à un contrôle façonné « par les priorités économiques et extractives dans les années 1930 » (p. 281). L'auteure commence par placer les entreprises soviétiques dans un cadre global. Elle perçoit ainsi des politiques de « management environnemental » similaires à l'Est et à l'Ouest dans les années 1930. Des mesures d'urgence du temps de guerre au début des années 1920, les spécialistes soviétiques de santé publique et de médecine tropicale passent à des politiques de " contrôle du vecteur » visant à endiguer la malaria notamment par des « travaux hydrotechniques » (p. 288) dans « une approche complète de la modification de l'environnement naturel », mais aussi des travaux d'instruction « introduisant la médecine européenne » dans la région (p. 290). Walker perçoit le tournant dans la lutte contre la malaria par une « combinaison de bureaucratisation et une réalité tadjike pauvre en ressources » (p. 294) Avec la collectivisation, les spécialistes perçoivent la malaria à travers le prisme d'une quantification détaillée des pertes économiques dues à la maladie (p. 294).

Douglas Weiner et John Brooke concluent l'ouvrage en replaçant l'histoire de la Russie eurasienne dans une perspective globale de longue durée. Adoptant le cadre du « projet développementaliste » de Kenneth Pomeranz, ils voient la diffusion des empires russe et soviétique à travers l'Eurasie et « leur histoire d'extraction destructrice » comme « une pièce d'un même schéma global commençant au début de la période moderne, et infléchi par des contextes spécifiques » (p. 299). Les deux auteurs mettent en avant combien « l'intelligentsia russe et l'État russe ont travaillé ensemble pour créer des Néo-Russies - des sous-ensembles des Néo-Europes -, mais [comment] à côté de cela, ils créèrent des territoires qui ressemblaient fortement aux dépendances coloniales des autres empires » (p. 300). Après avoir dégagé les traits transversaux des contributions de l'ouvrage qui permettent de rapprocher l'histoire environnementale des Empires russe et soviétique des problématiques de l'histoire environnementale moderne, les deux auteurs concluent toutefois que la « construction de l'empire russe a eu lieu dans des conditions environnementales plus rudes et difficiles que dans les Amériques tempérées » (p. 313). D'abord les colonisateurs russes n'ont pas provoqué le choc épidémiologique favorisant la conquête décrite par Crosby. Ensuite, « le potentiel agricole des territoires » conquis était plus faible du fait du « manque de précipitations, et d'étendues désertiques et montagneuses » (p. 313). Enfin, contrairement à l'Amérique du Nord-Est, « les défis écologiques et indigènes rendirent la construction impériale bien plus difficile ». Ces conditions ont forcé les États à des « comportements court-termistes de prélèvements de tributs ». Les régimes tsariste et soviétique ont dû « exploiter leurs 'autres' intérieurs, principalement les paysanneries russe et ukrainienne » (p. 314). Pour Weiner et Brooke, les cas d'étude du 
volume incitent à un rejet de l'exceptionnalisme russe et soviétique, « nous délivrant ainsi des entraves historiographiques qui ont pesé sur notre compréhension de la guerre de Crimée jusqu'à la guerre froide et au-delà » (p. 315).

Eurasian Environments est un ouvrage fondamental. Il représente une étape dans le décloisonnement amorcé relativement récemment entre les champs de recherche de l'histoire environnementale d'un côté et des régions eurasiatiques de l'autre. Si des comparaisons et des connexions transnationales ont été tracées ici et là, on attend toutefois encore un déploiement plus poussé des études tout à la fois particulières et « globales » (transnationales, connectées, imbriquées, circulatoires, etc.) auxquelles nous incitent les défis écologiques actuels. Par ailleurs, si l'histoire environnementale porte depuis ses origines un caractère intrinsèquement transdisciplinaire, les approches franchissant pleinement les frontières internes des sciences humaines et sociales - qui fleurissent aujourd'hui sous le sceau des « humanités environnementales »-, mais aussi celles des sciences « dures », sont encore relativement peu représentées dans la recherche sur ces aires géographiques. Eurasian Environments devrait ainsi constituer à la fois une base historiographique solide et un terreau fertile pour le développement de recherches intégrant pleinement les localités de la Russie eurasiatique à une histoire environnementale mondiale en prise avec les enjeux écologiques et sociétaux présents et à venir. Espérons que ces enquêtes seront autant que possible multiples dans leurs méthodologies, leurs approches et leurs perspectives. 